\title{
Testing psychological theories about inference making
}

\author{
ALAN GARNHAM \\ Centre for Research on Perception and Cognition, Laboratory of Experimental Psychology \\ University of Sussex, Brighton BNI 9QG, England
}

\begin{abstract}
Two contrasting views about elaborative inferences in text comprehension are that they occur as the text is read and that they are deferred until they are made necessary by a question about the inferable information. This paper develops these views in greater detail and examines some of their consequences. The model of memory performance underlying the first of these theories has two quite different interpretations: the standard one and one in which inferable information, whether it is implicit or explicit in a text, is not encoded into memory for content. Two experiments are reported. The results are not consistent with the most straightforward interpretation of the claim that inferences are always deferred. However, that theory can be modified so that it makes the same predictions as its rivals. The various interpretations of the formal model underlying all three accounts of inference making can be distinguished only in terms of parsimony, explanatory adequacy, and their ability to handle results from other experimental paradigms, such as sentence verification.
\end{abstract}

Two kinds of inference can be made in understanding a text: those that are necessary for integrating information from its various sentences into a coherent overall representation of its content, and those that are merely elaborative. There is little doubt that necessary inferences are made "on-line." For example, readers quickly determine the referents of anaphoric pronouns, even when quite elaborate inferences are needed, as in "Sue sold her car to Pam because she had decided to take up cycling," and experimental evidence shows that bridging inferences (Clark, 1977), which are also necessary to establish coherence, occur as text is read (Gamham, 1981; Garrod \& Sanford, 1977; Haviland \& Clark, 1974).

But what of elaborative inferences? Psychologists have failed to agree on whether they are also made online or whether they are deferred and made only if they are needed. On one hand, experiments using a variety of memory paradigms have provided indirect evidence for the immediate encoding of inferences (the immediate inference theory). People confuse sentences they have seen with sentences inferable from them. On the other hand, a number of sentence verification experiments, mainly by Singer $(1979,1980,1981)$, suggest that

The experiments reported in this paper were part of a doctoral thesis submitted to Sussex University. The work was funded by a grant from what was then the Science Research Council (Great Britain). The theoretical ideas in the paper were worked out while the author held a further grant from the Science and Engineering Research Council (Great Britain). Phil Johnson-Laird provided many useful comments on previous drafts. Chuck Clifton and two anonymous reviewers made a number of invaluable suggestions concerning the presentation of the models and the analysis of the data. inferences are not made on-line. For example, Singer (1979) showed that subjects verified "The sailor swept the floor with the broom" faster when they had just been presented with "The sailor used the broom to sweep the floor," which explicitly mentions the broom as the instrument used in sweeping, than when they had just seen "The sailor swept the floor in the cabin." According to this deferred inference theory, inferences will be made only if they are required for answering specific questions.

Neither theory receives strong empirical support. Singer's results provide only weak evidence for the postponement of inferences, because there is greater lexical overlap between the originally presented sentences and the to-be-verified sentences in conditions in which verification is found to be quicker. And arguing from memory studies to on-line processes requires assumptions that are not independently motivated. Only if the principle of encoding specificity (Tulving \& Thomson, 1973) applied to the mental representation of text could confusions in memory protocols be taken as a strong indication of what happened as those texts were read. Furthermore, Corbett and Dosher (1978) have used memory data to argue against the immediate inference theory. They showed that not only is "grocer shovel" a good recall cue for both "The grocer dug the hole" and "The grocer dug the hole with the shovel," but it is also a good cue for "The grocer dug the hole with a pitchfork" (better than "grocer pitchfork"). Thus, the fact that a highly probable instrument is a good cue for a sentence does not imply that the instrument has been encoded in the representation of the sentence.

Much of the trouble in deciding between the two theories can be attributed to their imprecision. In what 
follows, their predictions will be worked out in more detail, particularly for experiments that compare the three kinds of material used by Corbett and Dosher (1978): one in which the subject can draw an inference, one in which the most probable result of that inference is explicitly stated, and one in which that probable result is overridden by explicit information to the contrary. The formalization of the theories brings a number of in teresting points to light.

In order to derive the predictions, two assumptions will be made, common to both theories and justifiable on independent grounds. First, every text receives two encodings, one of content and one of surface form (Dooling \& Christiaansen, 1977; Johnson-Laird \& Stevenson, 1970). Second, when memory traces decay, reconstructive processes may be able to determine what has most probably been lost from them. Such processes work on what remains of the representations, and they make use of general knowledge about the world.

Consider an experiment in which subjects are asked to remember whether explicit case fillers (e.g., instruments or agents) occurred at certain points in a text that they have previously heard. To answer correctly, they should base their responses on a representation into which no inferences have been encoded. Representations of surface form do not incorporate inferences, but they decay fairly rapidly. The deferred inference theory claims that representations of content always accurately reflect what was presented, but according to the immediate inference theory, case fillers implicit in the text become explicit in this representation. Thus a representation of content that contains inferable information may correspond to an implicit or an explicit case filler in the original sentence. The immediate inference theory, therefore, predicts that responses based on memory traces will tend to be less accurate for implicit and highly probable case fillers than for improbable ones. When a highly probable filler is encoded, subjects will have to guess whether it was originally presented.

Similarly, a guessing mechanism must be invoked when reconstructive processes generate highly probable case fillers, because such fillers are only sometimes explicitly mentioned in texts. An estimate must be made of the likelihood that a reconstructed case filler was explicitly mentioned. The number of sentences in each of the experimental conditions will probably affect this estimate. Reconstructive processes do not generate improbable case fillers, and, therefore, they increase the relative proportions of "implicit" and "highly probable" responses in the tests.

At the time of testing, let $s$ be the probability that surface form still correctly reflects the kind of instrument presented and let $c$ be the probability that content does. Let $\mathrm{q}$ be the probability that a highly probable case filler, from a source that is not a reliable guide to what was presented, is omitted in the memory protocol. Such guessing occurs in two cases: when the case filler has been reconstructed and, in the immediate inference theory, when it is present in the representation of content and surface form has decayed. Tables 1 and 2 show the predictions of the immediate inference and deferred inference theories, respectively. ${ }^{1}$ In the last column of Table 1 and all the columns of Table 2 , the probability that a correct response can be based on memory traces is $s+c(1-s)$. It is assumed that the two memory traces are independent. Reconstruction followed by guessing comes into play in the other $(1-s)(1-c)$ cases. However, in the first two columns of Table 1, memory traces are useful only in a proportion $s$ of cases, since content is an unreliable guide to what was presented and guessing is required in $1-s$ cases.

Table 1

Predicted Proportions of Remembered Case Fillers for Each Kind of Presented Case Filler According to the Immediate Inference Theory

\begin{tabular}{|c|c|c|c|}
\hline \multirow[b]{2}{*}{ Remembered } & \multicolumn{3}{|c|}{ Presented Case Filler } \\
\hline & None & Highly Probable & Improbable \\
\hline $\begin{array}{l}\text { None } \\
\text { Highly Improbable } \\
\text { Improbable }\end{array}$ & $\begin{array}{l}s+q(1-s) \\
(1-q)(1-s) \\
0\end{array}$ & $\begin{array}{l}q(1-s) \\
s+(1-q)(1-s) \\
0\end{array}$ & $\begin{array}{l}q(1-s)(1-c) \\
(1-q)(1-s)(1-c) \\
s+(1-s) c\end{array}$ \\
\hline
\end{tabular}

Note-Column totals must be 1 .

Table 2

Predicted Proportions of Remembered Case Fillers for Each Kind of Presented Case Filler According to the Deferred Inference Theory

\begin{tabular}{llcc} 
& \multicolumn{3}{c}{ Presented Case Filler } \\
\cline { 2 - 4 } \multicolumn{1}{c}{ Remembered } & \multicolumn{1}{c}{ None } & Highly Probable & Improbable \\
\hline None & $s+(1-s)[c+(1-c) q]$ & $(1-s)(1-c) q$ & $(1-s)(1-c) q$ \\
Highly Probable & $(1-s)(1-c)(1-q)$ & $s+(1-s)[c+(1-c)(1-q)]$ & $(1-s)(1-c)(1-q)$ \\
Improbable & 0 & 0 & $s+(1-s) c$ \\
\hline
\end{tabular}

Note-Column totals must be 1 . 
Consider the middle entry in Table $2, s+(1-s)$ $[c+(1-c)(1-q)]$, the predicted proportion of "highly probable" responses, given that a highly probable explicit case filler occurred in the text. According to the deferred inference theory, the representations of both surface form and content are accurate guides to whether a probable case filler was presented. The first term in the expression, $s$, represents the probability of being able to make a correct response on the basis of surface form. In a proportion $1-\mathrm{s}$ of the cases, surface form has decayed and therefore it does not specify the presented case filler. In a proportion $\mathrm{c}$ of these cases, content is still intact and provides the correct answer. In the remaining $(1-s)(1-c)$ cases, neither memory trace specifies the presented case filler, and the most probable one must be reconstructed. In a proportion $1-q$ of these cases, the subjects guess that a highly probable case filler was actually presented. Thus the proportion of "highly probable" responses is effectively increased from $s+c-s c$, the probability of a correct response based on accurate memory traces, by $(1-q)$ $(1-s)(1-c)$.

Table 1 reveals an important fact about the immediate inference theory. Although the model was derived from the assumption that inferences are made on-line, its predictions depend only on the assumption that memory for content is of no use in deciding whether a highly probable case filler was actually presented. One explanation of this fact could be that such case fillers are encoded into the representation even if they are only implicit, but another is that they are never encoded. The latter theory, which might be called the omission theory, claims that highly probable case fillers whether explicit or implicit are left out of representations of content. If it is ever necessary to know what a case filler was, for example to answer a question about an implicit instrument or agent, it can be reconstructed from general knowledge and the context of the passage. Improbable case fillers, unlike probable ones, must be encoded, because they cannot be reconstructed. As the roles of surface form (s), content (c), and guessing (q) are the same in the omission theory as in the immediate inference theory, the predictions of the omission theory are identical to those in Table 1.

It may appear from Tables 1 and 2 that the immediate and deferred inference theories have the same power, since both models have three parameters. However, in
Table 2 there are only two effective parameters. The reason is quite straightforward. According to the deferred inference theory, neither content nor surface form is differentially effective as a cue to what was presented, for the three kinds of material. Its model, therefore, only contains a single "memory" parameter, $\mathrm{t}=\mathrm{s}+\mathrm{c}-\mathrm{sc}$, which reflects the probability that information about the presented case filler is still encoded. Thus Table 2 can be recast as Table 3 .

A number of specific predictions follow from Tables 1 and 3 , reflecting underlying principles of the theories. The deferred inference theory predicts that performance should be better with implicit and highly probable case fillers than with improbable ones. The reason is that memory traces are equally useful for remembering all kinds of case fillers, but reconstruction increases the number of "implicit" and "highly probable" responses. The theory also predicts that subjects will claim that no case filler was presented equally often when highly probable and improbable ones actually were presented, as the guessing mechanism is invoked in the same proportion, $1-t$, of the cases when the memory traces have decayed. On the other hand, the immediate inference and omission theories make no prediction about the relative proportions of correct responses for the three kinds of case fillers. They predict that the number of false alarms to implicit case fillers should be greater when highly probable ones were presented than when improbable ones were, since $(1-c)<1$.

These predictions can be used to perform detailed experimental tests between two classes of model: one that claims representations of content provide information about the occurrence of highly probable case fillers, and one that claims they do not.

\section{EXPERIMENT 1}

This experiment investigates the encoding of implicit and explicit agents. A continuous text was presented that described a series of actions for which there were highly probable and improbable agents. It is reasonable to assume that people have some knowledge about the relative probabilities of different possible agents for actions, taking into account the context in which those actions occur. For example, consider the sentence "They were surprised to find that the rubbish had not been collected." Household refuse is typically collected

Table 3

Predicted Proportions of Remembered Case Fillers for Each Kind of Presented Case Filler According to the Deferred Inference Theory (Two-Parameter Formulation)

\begin{tabular}{llll} 
& \multicolumn{3}{c}{ Presented Case Filler } \\
\cline { 2 - 4 } Remembered & \multicolumn{1}{c}{ None } & Highly Probable & Improbable \\
\hline None & $\mathrm{t}+\mathrm{q}(1-\mathrm{t})$ & $\mathrm{q}(1-\mathrm{t})$ & $\mathrm{q}(1-\mathrm{t})$ \\
Highly Probable & $(1-\mathrm{q})(1-\mathrm{t})$ & $\mathrm{t}+(1-\mathrm{q})(1-\mathrm{t})$ & $(1-\mathrm{q})(1-\mathrm{t})$ \\
Improbable & 0 & 0 & $\mathrm{t}$ \\
\hline
\end{tabular}

Note-Column totals must be 1. 
by dustmen (in England). So if a highly probable agent were to be mentioned in the above sentence, the phrase "by the dustmen" would probably appear in it. If, instead, the phrase "by the army" occurred, it would indicate a possible, but not very probable, agent for the action. The passage was constructed so that it was never necessary to encode a particular agent in order to understand the subsequent sentences in it, so inferences to agents were always merely elaborative. The encoding of the passage was examined in recall and recognition tests, in order to test the theories outlined above.

\section{Method}

Subjects. Twenty subjects took part in this experiment as part of a 2nd-year psychology course at Sussex University. They were not paid. The experiment lasted about $45 \mathrm{~min}$.

Materials. A passage about a mother's trip to the dentist was written (see Appendix A). Thirty clauses in the passage, describing various actions, were in the passive voice: for example, the second clause in the sentence "Mr. Brown had a letter to say that their old house had been sold." Accordingly, these clauses made sense either with or without a phrase such as "by the estateagent," which indicates the agent of the action. Of the 30 clauses selected, 10 had no agent mentioned explicitly (e.g., "The milkman had been bitten as he delivered the milk."), 10 had highly probable agents explicitly mentioned (e.g., "At the till she was short-changed by the cashier."), and 10 had improbable agents explicitly mentioned (e.g., "A child had been knocked down by a traction-engine."). The passage was recorded on a Revox tape recorder by a male native speaker of English.

The memory tests used typed versions of the passage. There was one recall test and one recognition test. Each test had 42 items in it, 1 related to each of the 30 selected actions, and 12 filler items, included to divert subjects' attention from the structure of the tests.

In the recall tests 42 sets of empty parentheses [i.e.,"( )"], appeared at various points throughout the passage. Thirty occurred at places where phrases mentioning the agents of the selected actions were located or could have been located in the passage. The other 12 occurred at places where arbitrarily selected nouns and temporal expressions were omitted from the passage.

The recognition test was identical to the recall test, except that the 42 sets of parentheses each contained three phrases, for example, "(by a dog/by a fly/neither of these)." In the case of the 30 tests related to the selected actions, the first two phrases in the parentheses were those mentioning the highly probable and the improbable agents. For the filler tests, they were the phrase that had been omitted from the passage and another phrase that could sensibly occur at the same point in the passage. For each item in the recognition test, the order of the first two phrases was randomized independently. The third item was always the phrase "neither of these." This was the correct answer when the presented clause of the passage had an implicit agent.

Design. All subjects heard the same passage in presentation. Accordingly, the various kinds of clauses mentioning agents (no agent, highly probable agent, improbable agent) constituted a within-subjects between-materials variable. Each subject heard 10 clauses of each kind, but any particular sentence occurred in only one form.

Procedure. The subjects were tested in a group and heard the passage over a single loudspeaker. Before they heard it, they were told to listen carefully, as they would be asked questions about it later.

After they had heard the passage, subjects were given an interpolated task, to produce some forgetting and to eliminate recall from short-term memory. The task was to work through a typewritten passage crossing out all occurrences of the letter "e." Four minutes were spent on the task itself, but with delays caused by handing out and giving in papers, the time between presentation and the first memory test was about $10 \mathrm{~min}$.

The recall test was given first. If subjects had previously seen the recognition test, the items in the parentheses might have affected their performance in the recall test. Subjects were asked to write inside each set of parentheses the word or words, if any, that had been omitted from the passage at the place where the parentheses occurred. They were told to leave the parentheses blank if no word(s) had been omitted. No time limit was imposed, but subjects were asked not to go back and change their responses while they were waiting for other people to finish. The instructions encouraged subjects to produce the gist of the omitted material if they could not remember the exact words they had heard.

The recognition test followed the recall test immediately. Subjects had to underline the alternative in each set of parentheses that had occurred in the passage. The alternative "neither of these" was to be chosen if (1) no word or phrase had been omitted from the passage or (2) a word or phrase had been omitted, but it was neither of the first two alternatives in the parentheses. Only the first of these was ever a correct reason for choosing "neither of these"; the second reason was included in the instructions to justify the presence of "neither of these" in the filler tests. "Neither of these" was required in the filler tests so that all the test items looked the same.

Scoring procedure. In the recall test, synonyms, superordinates, and close cohyponyms were counted as correct. When the agents were implicit or highly probable, the subjects could not be expected to know, when completing the recall test, which improbable agent occurred in the recognition test. Recall of any improbable agent was therefore counted as a confusion.

\section{Results}

Recall. Table 4 summarizes the responses given in the recall test as a function of the kind of agent presented. The models in Tables 1 and 3 were fitted to the data by the method of least squares. The fourth row of Table 4 (i.e., "Other Responses") was ignored for the purposes of fitting the models. These models accounted for 99.5\% ( $\hat{\mathrm{s}}=.5573, \hat{\mathrm{c}}=.5261, \hat{\mathrm{q}}=.6282)$ and $96.4 \%$ $(\hat{\mathrm{t}}=.6979, \hat{\mathrm{q}}=.6655)$ of the variance in Table 4 , respectively. Thus, both models fitted the data well, with the third parameter in the model of Table 1 accounting for only a small percentage of the variance. However, focused comparisons, derived from the predictions outlined in the introduction, showed that the deferred inference theory was not consistent with the data. Subjects were more likely to omit highly probable agents than improbable ones $(51$ vs. 27$)[\mathrm{t}(19)=3.48, \mathrm{p}<.005$,

Table 4

Number of Responses of the Various Kinds in the Recall Test in Experiment 1

\begin{tabular}{lrrrr}
\hline & \multicolumn{4}{c}{ Type of Agent Presented } \\
\cline { 2 - 5 } $\begin{array}{l}\text { Type of Agent } \\
\text { Recalled }\end{array}$ & None & $\begin{array}{c}\text { Highly } \\
\text { Probable }\end{array}$ & $\begin{array}{c}\text { Improb- } \\
\text { able }\end{array}$ & Total \\
\hline None & 153 & 51 & 27 & 231 \\
Highly Probable & 21 & 141 & 9 & 171 \\
Improbable & 0 & 0 & 156 & 156 \\
Other Responses & 26 & 8 & 8 & 42 \\
Total & 200 & 200 & 200 & 600 \\
\hline
\end{tabular}


Table 5

Number of Responses of the Various Kinds in the Recognition Test in Experiment 1

\begin{tabular}{lrccc}
\hline & \multicolumn{4}{c}{ Type of Agent Presented } \\
\cline { 2 - 5 } $\begin{array}{c}\text { Type of Agent } \\
\text { Recognized }\end{array}$ & None & $\begin{array}{c}\text { Highly } \\
\text { Probable }\end{array}$ & $\begin{array}{c}\text { Improb- } \\
\text { able }\end{array}$ & Total \\
\hline None & 110 & 22 & 12 & 144 \\
Highly Probable & 83 & 174 & 13 & 270 \\
Improbable & 7 & 4 & 175 & 186 \\
Total & 200 & 200 & 200 & 600 \\
\hline
\end{tabular}

one-tailed], and improbable agents were more often remembered correctly than highly probable ones (156 vs. 141) $[\mathrm{t}(19)=1.99, \mathrm{p}<.05$, one-tailed $]$. In other words, the likelihood that improbable agents were more difficult to recall than highly probable ones, as predicted by the deferred inference theory, was less than $5 \%$.

Recognition. Table 5 summarizes the responses given in the recognition test, as a function of the kind of agent presented. The two models accounted for $99.7 \%(\hat{\mathrm{s}}=.4442, \hat{\mathrm{c}}=.7942, \hat{\mathrm{q}}=.2242)$ and $87.0 \%$ $(\hat{\mathrm{t}}=.7039, \hat{\mathrm{q}}=.1454)$ of the variance, respectively.

Subjects claimed that no agent had been presented when a highly probable one had been more frequently than they did with improbable agents (22 vs. 12) [t(19) $=2.07, \mathrm{p}<.05$, one-tailed $]$. Although there was no significant difference in the number of correctly recognized highly probable and improbable agents (174 vs. $175)$, subjects were much better at recognizing that an improbable agent had been presented than at recognizing that none had been $(175$ vs. 110$)\lceil t(19)=6.08$, $\mathrm{p}<.001$, one-tailed] .

\section{Discussion}

In both recognition and recall, subjects were more likely to omit a highly probable agent than an improbable one. In neither test were improbable agents the most difficult to remember correctly. These findings disconfirm the deferred inference theory. The first of these findings is predicted by the immediate inference and omission theories, and the second is compatible with them (see Tables 1 and 3).

In Experiment 1, very few of the effects reached significance on a by-materials analysis (Mann-Whitney $\mathrm{U}$ tests). The lack of significance was probably attributable to the small number of materials (10) in each condition. In the second experiment, a different experimental design was used, in an attempt to overcome this problem.

\section{EXPERIMENT 2}

This experiment investigates the encoding of implicit and explicit instruments. A continuous text was presented that described a series of actions for which there were highly probable and improbable instruments. For example, for the action described in the sentence "The two boys landed on the island and tied up their vessel," a piece of rope is a highly probable instrument, whereas a strip torn off an old sheet is rather unlikely.

The experiment is similar in conception and form to Experiment 1, except that a more satisfactory withinmaterials design was used. In different versions of the passage, each test sentence occurred in three different forms: with no instrument explicitly mentioned, with a highly probable instrument mentioned, and with an improbable instrument mentioned.

A further manipulation in the second experiment was that half of the subjects were given incidental learning instructions and the rest, intentional. Despite the well established finding that intentional instructions tend to produce better verbatim memory (e.g., Johnson-Laird \& Stevenson, 1970), this variable had no effects in the present experiment and will not be mentioned further.

\section{Method}

Subjects. The 12 subjects were volunteers from the student population of Sussex University. They were paid 60 pence for participating in the experiment, which lasted about $30 \mathrm{~min}$. None of the subjects from Experiment 1 took part in the present experiment.

Materials. A passage about workmen on a worksite was written (see Appendix B). Twenty-four actions were described in the passage that required instruments for their performance. For example, when workmen drive to a worksite, they must have some vehicle (e.g., a minibus) to take them. That vehicle is instrumental in their driving to the worksite.

The passage was constructed so that it made sense both when the phrases referring to instruments were included and when they were not. There were, accordingly, three versions of each of the 24 clauses describing the selected actions, for example, "The men were to drive to the worksite," "The men were to drive to the worksite in a minibus," and "The men were to drive to the worksite on motorbikes." In order that all three versions of each sentence could be used, three versions of the passage were constructed. They were recorded on a B\&O tape recorder, with the author as speaker. The memory tests were similar to those in Experiment 1. There were 34 items in each of the tests, 24 related to the chosen actions, and 10 fillers, included to divert subjects' attention from the structure of the test (see Experiment 1, "Materials," for details of how the tests were derived from the passage).

Design. There were eight actions of each kind in each version of the passage. Kind of instrument was both a within-subjects and a within-materials variable.

Procedure. Subjects were tested individually. They listened to one of the versions of the passage in a small quiet room. When they had heard the passage, the subjects were given an interpolated task, which comprised 40 three-digit number plus threedigit number addition problems (eg., $573+635=$ ?). Subjects had $10 \mathrm{~min}$ to do this task. If they finished all the problems before $10 \mathrm{~min}$ had elapsed, they moved onto the memory tests immediately. ${ }^{2}$ The memory tests were administered as in Experiment 1 .

\section{Results}

Recall. Table 6 shows the number of responses of different kinds in the recall test, as a function of the kind of instrument presented. The models in Tables 1 and 3 accounted for $98.9 \%(\hat{\mathrm{s}}=.3630, \hat{\mathrm{c}}=.4755, \hat{\mathrm{q}}=$ 
Table 6

Number of Responses of the Various Kinds in the Recall Test of Experiment 2

\begin{tabular}{lcccc}
\hline & \multicolumn{4}{c}{ Type of Instrument Presented } \\
\cline { 2 - 5 } $\begin{array}{c}\text { Type of Instrument } \\
\text { Recalled }\end{array}$ & None & $\begin{array}{c}\text { Highly } \\
\text { Probable }\end{array}$ & $\begin{array}{c}\text { Improb- } \\
\text { able }\end{array}$ & Total \\
\hline None & 71 & 39 & 25 & 135 \\
Highly Probable & 14 & 50 & 4 & 68 \\
Improbable & 1 & 0 & 64 & 65 \\
Other Responses & 10 & 7 & 3 & 20 \\
Total & 96 & 96 & 96 & 288 \\
\hline
\end{tabular}

Table 7

Number of Responses of the Various Kinds in the Recognition Test of Experiment 2

\begin{tabular}{|c|c|c|c|c|}
\hline \multirow[b]{2}{*}{$\begin{array}{l}\text { Type of Instrument } \\
\text { Recognized }\end{array}$} & \multicolumn{4}{|c|}{ Type of Instrument Presented } \\
\hline & None & $\begin{array}{c}\text { Highly } \\
\text { Probable }\end{array}$ & $\begin{array}{l}\text { Improb- } \\
\text { able }\end{array}$ & Total \\
\hline None & 55 & 25 & 15 & 95 \\
\hline Highly Probable & 38 & 68 & 9 & 115 \\
\hline Improbable & 3 & 3 & 72 & 78 \\
\hline Total & 96 & 96 & 96 & 288 \\
\hline
\end{tabular}

$.7049)$ and $91.6 \%(\hat{\mathrm{t}}=.5479, \hat{\mathrm{q}}=.7572)$ of the variance, respectively. The overall pattern of results was very similar to that obtained in Experiment 1. Subjects omitted highly probable instruments more frequently than improbable ones ( 39 vs. 25$)[\mathrm{t}(11)=3.63, \mathrm{p}<.005$, one-tailed]. This effect also held good by materials $[\mathrm{t}(23)=1.77, \mathrm{p}<.05$, one-tailed $]$. Improbable instruments were more reliably remembered than highly probable ones (64 vs. 50) [by-subjects $t(11)=5.63$, $\mathrm{p}<.0005$, one-tailed; by-materials $\mathrm{t}(23)=1.80, \mathrm{p}<.05$, one-tailed].

Recognition. Table 7 summarizes responses in the recognition tests, as a function of the kind of instrument presented. The models accounted for $98.9 \%$ $(\hat{\mathrm{s}}=.3094, \hat{\mathrm{c}}=.6465, \hat{\mathrm{q}}=.4153)$ and $82.8 \%(\hat{\mathrm{t}}=.5750$, $\hat{\mathrm{q}}=.4183$ ) of the variance, respectively, Again, overall performance was very similar to that found in Experiment 1 . Highly probable instruments were more likely to produce "neither of these" responses than were improbable ones (25 vs. 15) [by-subjects $t(11)=2.42$, $\mathrm{p}<.025$; by-materials $\mathrm{t}(23)=2.32, \mathrm{p}<.025$, onetailed]. Subjects found it easier to recognize improbable instruments than to recognize that no instrument had been presented (72 vs. 55) [by-subjects $t(11)=2.05$, $\mathrm{p}<.05$; by-materials $\mathrm{t}(23)=2.10, \mathrm{p}<.05$, one-tailed $]$.

\section{Discussion}

The results of the present experiment are in all respects very similar to those of Experiment 1 and, therefore, corroborate the findings of that experiment.

\section{GENERAL DISCUSSION}

Both of the experiments provide evidence that is consistent with the predictions of the immediate inference and omission theories, but not with those of the deferred inference theory. However, it is not really surprising that a three-parameter model can give a better account of a set of data than a two-parameter model can. A third parameter can readily be incorporated into the deferred inference theory, although the most plausible ways of doing so violate the spirit of the theory. For example, it could be assumed that the memory traces (content or surface form or both) are inherently more reliable for improbable case fillers than for highly probable and implicit ones. This assumption entails that highly probable and improbable case fillers are differently represented, and it is difficult to see how this state of affairs could arise unless they are treated differently at encoding. This elaboration of the deferred inference theory, therefore, sacrifices one of its basic assumptions. Let the probabilities of the two kinds of case filler being reliably represented at the time of testing be $i$ and $p$, respectively, with $\mathrm{i}<\mathrm{p}$. The predictions of this revised version of the deferred inference theory are shown in Table 8. It is quite a simple matter to show that Table 8 is equivalent to Table 1 , with $p=s$ and $i=s+c-s c$. Thus, three apparently very different theories about inference making in text comprehension, the immediate inference theory, the omission theory, and a revised version of the deferred inference theory, make the same predictions for memory experiments. There remain two ways of deciding among them: (1) considerations of parsimony, plausibility, and explanatory power and (2) ability to account for results from other experimental paradigms.

The immediate inference theory has two major failings. First, it is not consistent with the results of the sentence verification experiments mentioned in the introduction. Although those experiments were method-

Table 8

Predicted Proportions of Remembered Case Fillers for Each Kind of Presented Case Filler According to the Deferred Inference Theory (Revised Formulation)

\begin{tabular}{llll}
\hline & \multicolumn{3}{c}{ Presented Case Filler } \\
\cline { 2 - 4 } Remembered & \multicolumn{1}{c}{ None } & Highly Probable & Improbable \\
\hline None & $\mathrm{p}+\mathrm{q}(1-\mathrm{p})$ & $\mathrm{q}(1-\mathrm{p})$ & $\mathrm{q}(1-\mathrm{i})$ \\
Highly Probable & $(1-\mathrm{q})(1-\mathrm{p})$ & $\mathrm{p}+(1-\mathrm{q})(1-\mathrm{p})$ & $(1-\mathrm{q})(1-\mathrm{i})$ \\
Improbable & 0 & 0 & $\mathrm{i}$ \\
\hline
\end{tabular}

Note-Column totals must be 1 . 
ologically flawed, the effects obtained were rather large to be explained by lexical priming, so the results at least suggest that inferences are not encoded as text is read. Second, the formal development of the immediate inference theory shows it to be implausible. It claims that text processing is inefficient in that inferred information is stored even though it can be reconstructed from other information in the representation.

The deferred inference theory is able to accommodate the results of the sentence verification experiments, but it lacks explanatory power in accounting for the memory data. There was no good theoretical motivation for the introduction of the third parameter into its model. It was simply added post hoc, to account for the experimental findings, and its interpretation was not really consistent with the original theory. The three parameters of the immediate inference and the omission theories are, on the other hand, independently motivated. Those theories, therefore, provide a more principled account of why there tends to be a relatively large proportion of "improbable" responses in memory protocols. In the omission theory, in particular, the effect is a result of efficient encoding. There are indefinitely many inferences that can be made from a text, and it would be very wasteful to store them all, particularly when they can be derived from information that must be encoded.

The omission theory is also able to reconcile the results of the memory and sentence verification experiments. Memory for surface form is known to be relatively short-lived, so at long delays between presentation and testing, when responses reflect either memory for content or reconstruction, there will be many confusions between implicit and highly probable case fillers. Indeed, the response-bias parameter, q, can vary quite dramatically with the nature of the test, so that many positive responses can be obtained, even if none of the original sentences occur in the test.

In Singer's $(1979,1980,1981)$ sentence verification experiments, the delay between initial presentation and subsequent verification was short. Therefore, at the time of testing, memory for surface form was good and sentences that referred to previously explicit case fillers could be verified quickly. However, when the case filler is implicit, neither the representation of surface form nor that of content will encode it, and hence verification will be relatively slow. At longer delays, when responses must be based primarily on reconstruction from memory for content, whether the case filler was originally explicit or not, verification will always be slow and differences between the conditions should disappear. This result was obtained by Kintsch (1974, Chapter 8 ), although not specifically for case-filling inferences.

Of the three theories that could underlie the predictions for memory experiments in Table 1 , the omission theory is the most plausible. It is both compatible with results from other experimental paradigms, unlike the immediate inference theory, and able to give a parsimonious and explanatory account of behavior in memory experiments, unlike either the immediate inference theory or the deferred inference theory.

Although the present experiments have dealt only with inferences about instruments and agents, the omission theory generalizes to other kinds of inference. Our knowledge of the world is detailed and complex, we have a great deal of knowledge about relative probabilities, and whenever one state of affairs is the most likely one in a given context, it can be recovered from that context by reconstructive processes. It can be "remembered" even if it has not been encoded. However, the same mechanism cannot determine whether that state of affairs was explicitly mentioned or not.

Finally, when are inferences made? According to the omission theory, there is no simple answer to this question. Inferences result from processes that occur at encoding and retrieval, and the relative contribution of the two sets of processes depends on factors such as the delay between presentation and testing. Thus, encoding, storage, and retrieval are all implicated in inference making.

\section{REFERENCES}

Baddeley, A. D., \& Hitch, G. J. Recency re-examined. In S. Dornic (Ed.), Attention and performance VI. Hillsdale, N.J: Erlbaum, 1977.

Clark, H. H. Bridging. In P. N. Johnson-Laird \& P. C. Wason (Eds.), Thinking: Readings in cognitive science. Cambridge: Cambridge University Press, 1977.

Conbett, A. T., \& Dosher, B. A. Instrument inferences in sentence encoding. Journal of Verbal Learning and Verbal Behavior, $1978,17,479-491$.

Dooling, D. J., \& Christiansen, R. E. Levels of encoding and retention of prose. In G. H. Bower (Ed.), The psychology of learning and motivation (Vol. 11). New York: Academic Press, 1977.

Garnham, A. Anaphoric reference to instances, instantiated and non-instantiated categories: A reading time study. British Journal of Psychology, 1981, 72, 377-384.

GARrod, S., \& SANFord, A. J. Interpreting anaphoric relations: The integration of semantic information while reading. Journal of Verbal Learning and Verbal Behavior, 1977, 16, 77-90.

Haviland, S. E., \& ClaRK, H. H. What's new? Acquiring new information as a process of comprehension. Journal of Verbal Learning and Verbal Behavior, 1974, 13, 512-521.

Johnson-Laird, P. N., \& Stevenson, R. Memory for syntax. Nature, 1970, $227,412$.

KintsCH, W. The representation of meaning in memory. Hillsdale, N.J: Erlbaum, 1974.

Singer, M. The temporal locus of inference in the comprehension of brief passages: Recognizing and verifying implications about instruments. Perceptual and Motor Skills, 1979, 49, 185-201.

Sinoer, M. The role of case-filling inferences in the comprehension of brief passages. Discourse Processes, 1980, 3, 185-201.

Singer, M. Verifying the assertions and implications of language. Journal of Verbal Learning and Verbal Behavior, 1981, 20, 46-60.

Tulving, E., \& Thomson, D. M. Encoding specificity and retrieval processes in episodic memory. Psychological Review, 1973, 80, 352-373. 


\section{NOTES}

1. It is assumed that the decayed form of a memory trace that originally contained an instrument is never confused with the intact version of one that did not. The refutation of the deferred inference theory does not depend crucially on this assumption.

2. There is evidence to suggest that decay of short-term memory depends not on elapsed time, but on other tasks performed (see, e.g., Baddeley \& Hitch, 1977).

\section{APPENDIX A: PASSAGE USED IN EXPERIMENT 1}

Highly probable agents always precede improbable ones. Material in parentheses did not occur in the presented version of the passage.

"The Brown family were sitting down to breakfast (dinner). They had just opened the letters which had been delivered that morning (by the postman) by the village eccentric. Mr. Brown had a letter to say that their old house had been sold (by the estate-agent/by his brother) and a bill for a new hot-water tank (bath) which had been installed (by the plumber/by Mrs. Brown's cousin). The arrival of the post reminded him that he wanted to get a business letter typed that day by his secretary (by a temp) concerning a manuscript which he was anxious to have accepted (by a publisher) by a Japanese magazine. He also wanted to see about a new building which had been planned for his company's offices (social club) by an architect (by the mayor), and to decide whether they wanted a statue made (by a sculptor/by local schoolchildren) to stand in front of it. Mrs. Brown had a letter from her mother (great aunt) describing how the milkman had been bitten (by a dog/by a fly) as he delivered the milk, and how interesting the sermon preached last Sunday (the Sunday before last) (by the vicar) by the archbishop had been.

"That afternoon Mrs. Brown was going to have a tooth removed by the dentist (by a Frenchman). She had decided to spend the day in town, and to take the children with her. They got onto a bus (train), and their fares were collected by the conductor (by an inspector). Through the windows they could see that many of the fields had recently been ploughed (by the farmer) by the squire. As they came near to the town they passed a cricket match, and were just in time to see the ball hit for six (by the batsman/by a solicitor).

"Once they arrived in the town, they headed for the main shopping area. Mrs. Brown had several errands to do. First she ordered half a pig (sheep) for their deep-freezer. It was to be collected after the meat had been cut up (by the butcher/by the butcher's wife). Next she made an appointment to have her hair cut by the hairdresser (by an old friend of hers). Then she called to see if the suit (jacket) being made for her elder son (by the tailor/by the dressmaker) was finished, but it was not. Finally she took in a diamond (sapphire) ring which she wanted to have adjusted by the jeweller (by a man who did odd-jobs).

"Now that she had done these various errands, Mrs. Brown could go and do her shopping at the supermarket. At the till she was short-changed by the cashier (by the irritable girl), but the girl refused to admit she was wrong. Fortunately the mistake was sorted out by the manager (by a cleaner) when Mrs. Brown complained.

"As the family came out of the supermarket, they could see a large crowd gathering. A child had been knocked down (by a car) by a tractionengine and was being put onto a stretcher (by some ambulancemen) by some gardeners. Horns were being blown (by motorists) by idiots, but the cars could not move, since the traffic was being halted (by a policeman/by a bankclerk). Some weeks (days) later Mrs. Brown read in the local newspaper that the motorist had been found guilty of dangerous driving and sentenced to six month's (one year's) suspension from driving (by the magistrate/by the mayor's wife).

"For their lunch the family went to a restaurant which they particularly liked. They knew that the food prepared there by the chef (by a young German) was excellent. They ordered their meals, which were promptly brought in (by the waiter/by a Spaniard). During the meal, Mrs. Brown's little daughter threw some food on the floor, and she was scolded (by her mother) by an old woman.

"Mrs. Brown had not relished the prospect of the afternoon's visit to the dentist, and was glad that the extraction was not as painful as she expected. However, the dentist gave her a prescription and advised her to have it made up (by a chemist) by one of his acquaintances. The tablets would relieve any pain which might occur.

"When the family got home they were surprised to find that the rubbish had not been collected (by the dustmen) by the army, and that a dead mouse had been left on the kitchen floor by the cat (by the dog). When she saw the mouse, Mrs. Brown rushed into the kitchen, colliding with the door-frame (cooker). She broke a tooth, and had to be taken straight back to the dentist's."

\section{APPENDIX B: PASSAGE USED IN EXPERIMENT 2}

Highly probable instruments precede improbable ones. "The workmen were gathering in the company's yard. They were to drive to the worksite (in a minibus/on motorbikes). The foreman was thinking about (his brother/his mother), who had to fly to London that day (by plane/by helicopter). When all the men had arrived they set off. The journey lasted about (twenty minutes/an hour and a half). When they got there the foreman unlocked the door of the workmen's hut (with a key/with a piece of wire), and the men went inside to get their (equipment/jackets).

"Overnight a little earth had fallen into the hole they had been digging the previous day. One of the men dug it out (with a spade/with a pitchfork). While he was doing this the (waterpipes/ gas mains) which the men had to lay arrived from the company's yard (on a lorry/on a cart). The pipes were too heavy for the men to carry. They were lifted off the lorry (by crane/by a fork-lift truck) and taken to the hole.

"At $(10: 30 / 11: 15)$ the men had a tea break. John, whose turn it was to make the tea, went into the hut, washed his hands (with soap/with washing-up liquid), dried them (on a towel/on his shirt), and went to fetch the water. When he got back he lit the stove (with a match/with a cigarette lighter) so that he could boil the water (in the kettle/in the saucepan). As soon as the water boiled, John made the tea. He called to his mates and they came into the hut. The tea was poured out (into mugs/into jamjars) and sugar was stirred in (with a spoon/ with a pencil). One of the men had some extra (bread/cake) packed with his lunch (in his sandwich-box/in a shopping bag). As he was feeling hungry, he decided to eat some. He got it out and spread some butter on it (with a knife/with a penknife). Meanwhile the others were talking or reading (newspapers/ magazines). John saw an article which would interest his brother. He cut it out (with a pair of scissors/with a razor-blade).

"At the end of the tea-break the foreman remembered that he had to call up headquarters (on the phone/by radio-telephone). He was to be given the schedule of work for the next (week/ month). He banged on the table (with his fist/with a book) to get the men's attention, and told them that they would know what they were doing next week by lunchtime. The foreman wrote down the schedule (with a pencil/with a crayon) (on a piece of paper/on a scrap of cardboard). Then he prepared a 
notice and stuck it up on the notice-board (with a drawing$\mathrm{pin} /$ with glue).

"The worksite was near a park. As the men went out after their tea-break they could see two boys sailing on the lake (in a boat/in a bathtub). On the island in the lake (a boy and a girl/two girls) were trying to catch butterflies (in a net/in a paper-bag). The two boys landed on the island and tied up their vessel (with a piece of rope/with a strip torn off an old sheet). They stood an old (tin-can/cardboard box) on a rock, and tried to knock it off (with stones/with mudballs) from a distance."

(Received for publication February 2, 1982; revision accepted April 5, 1982.) 